\title{
Continuous Dunking Pancreaticojejunostomy: Our Experience with First Fifty Cases.
}

Ramesh Singh Bhandari, ${ }^{x}$ Paleswan Joshi Lakhey

Surgical Gastroenterology Units, Department of Surgery, T U Teaching Hospital

Email: rsbhandari09@gmail.com

\begin{abstract}
Introduction- Different techniques and modifications have been described to bring down the incidence of pancreatic fistula rate. However, no techniques have been accepted as a gold standard. Since 2011, we have been performing pancreaticojejunostomy by continuous dunking technique. Here, we intend to present our experience with the defined technique.
\end{abstract}

Methods: Prospectively maintained medical records of the patients (operated between Sept 2011 to Jan 2016) undergoing pancreaticoduodenectomy and pancreaticoenteric reconstruction by continuous dunking techniques were reviewed and analyzed. Postoperative complications mainly, incidence of post pancreatectomy fistula (POPF), hemorrhage (PPH) and delayed gastric emptying (DGE) along with other major complications were analyzed. Subgroup analysis was also performed to find the difference in fistula rate in patients with or without addition of Braun's anastomosis. ISGPS definition was used to define the major, surgery specific complications of pancreaticoduodenectomy.

Results: Total 51 patients underwent pancreaticojejunostomy with the defined technique with Male to female ratio of $23: 28$. Total $13.7 \%$ (7/51) patients received preoperative biliary drainage. Overall, clinically significant fistula rated was 15.6\% (8/51), PPH 13.7\% (7/51) and 0\% clinically significant DGE (overall DGE 13.7\%, 7/51). Mortality rate was $5.8 \%(3 / 51)$. Subgroup analysis performed between patients with or without addition of Braun's anastomosis revealed $0 \%$ clinically significant fistula rate with no mortality in the group.

Conclusion: Surgery specific complications following pancreaticoduodenectomy and pancreaticoenteric reconstruction with our technique are comparable to published results from high volume centers. Addition of Braun's anastomosis is a promising modification to bring down the POPF rate to a minimum.

Keywords: Pancreaticoduodenectomy, Pancreatic fistula, Braun's anastomosis 